\title{
Triplets of Spikes in a Model of Spike Timing-Dependent Plasticity
}

\author{
Jean-Pascal Pfister and Wulfram Gerstner \\ Laboratory of Computational Neuroscience, School of Computer and Communication Sciences and Brain-Mind Institute, Ecole Polytechnique Fédérale de \\ Lausanne, CH-1015 Lausanne, Switzerland
}

\begin{abstract}
Classical experiments on spike timing-dependent plasticity (STDP) use a protocol based on pairs of presynaptic and postsynaptic spikes repeated at a given frequency to induce synaptic potentiation or depression. Therefore, standard STDP models have expressed the weight change as a function of pairs of presynaptic and postsynaptic spike. Unfortunately, those paired-based STDP models cannot account for the dependence on the repetition frequency of the pairs of spike. Moreover, those STDP models cannot reproduce recent triplet and quadruplet experiments. Here, we examine a triplet rule (i.e., a rule which considers sets of three spikes, i.e., two pre and one post or one pre and two post) and compare it to classical pair-based STDP learning rules. With such a triplet rule, it is possible to fit experimental data from visual cortical slices as well as from hippocampal cultures. Moreover, when assuming stochastic spike trains, the triplet learning rule can be mapped to a Bienenstock-Cooper-Munro learning rule.
\end{abstract}

Key words: STDP; spike triplet; modeling; computational neuroscience; Hebbian learning; long-term potentiation

\section{Introduction}

During the last decade, an increasing number of experiments have shown that synaptic strength changes as a function of the precise spike timing of the presynaptic and postsynaptic neurons. In the early experiments (Markram et al., 1997; Bi and Poo, 1998, 2001; Zhang et al., 1998), potentiation has been elicited by a sequence of $n$ pairs of "pre then post" spikes, whereas depression occurred when the timing was reversed (i.e., when each postsynaptic spike precedes a presynaptic one). At this point, it was natural to characterize synaptic plasticity as a function of the time difference $\Delta t=t^{\text {post }}-t^{\text {pre }}$ between pairs of spikes. However, performing experiments with pairs of spikes does not mean that pairs of spikes are the elementary building block. There is no a priori reason to think that pairs of spikes are more relevant than three spikes (triplets), four spikes (quadruplets), or even more. It is clear that a lot of other neuronal variables such as calcium concentration (Malenka et al., 1988; Lisman, 1989; Lisman and Zhabotinsky, 2001; Shouval et al., 2002) or postsynaptic membrane potential (Rao and Sejnowski, 2001; Sjöström et al., 2001; Lisman and Spruston, 2005) play an important role in triggering potentiation or depression. The point of this study was to see how far we can explain experiments that only use spike timing as a parameter with models that only use spike timing.

Recent experiments (Bi and Wang, 2002; Froemke and Dan, 2002; Wang et al., 2005; Froemke et al., 2006) have studied the

\footnotetext{
Received April 4, 2006; revised Aug. 4, 2006; accepted Aug. 4, 2006.

This work was supported by the Swiss National Science Foundation 200020-108097. We gratefully acknowledge discussions with Taro Toyoizumi and Magnus Richardson.

Correspondence should be addressed to Prof. Wulfram Gerstner, Ecole Polytechnique Fédérale de Lausanne, Laboratory of Computational Neuroscience, Station 15, 1015 Lausanne, Switzerland. E-mail: wulfram. gerstner@epfl.ch.

DOI:10.1523/JNEUROSCI.1425-06.2006

Copyright $\odot 2006$ Society for Neuroscience $\quad 0270-6474 / 06 / 269673-10 \$ 15.00 / 0$
}

detailed role of spike timing by triggering synaptic plasticity with spike triplets (one presynaptic spike combined with two postsynaptic spikes or one postsynaptic spike with two presynaptic spikes). The results of those experiments indicate that classical spike timing-dependent plasticity (STDP) models based on pairs of spikes are not sufficient to explain synaptic changes triggered by triplets or quadruplets of spikes.

In the first part of this study, we review some experimental protocols performed in visual cortex (Sjöström et al., 2001) and hippocampal culture (Wang et al., 2005) and show why the classical pair-based STDP models fail to reproduce those experimental data. In the second part of this study, we show that if we assume that synaptic plasticity is governed by a suitable combination of pairs and triplets of spikes, the results from the above mentioned protocols can be surprisingly well reproduced. Moreover, we show that our triplet learning rule elicits input selectivity analogous to that of the Bienenstock-Cooper-Monro (BCM) theory (Bienenstock et al., 1982).

Claiming that triplet of spikes are more relevant than pairs of spikes is not enough to construct a model of synaptic plasticity. It is also necessary to determine how those pairs or triplets of spikes integrate. For both the pair-based models and the triplet-based models, we consider the case in which a presynaptic spike interacts with all previous postsynaptic ones or vice versa (we call this the All-to-All interaction) (Gerstner et al., 1996; Kempter et al., 1999; Kistler and van Hemmen, 2000; Song et al., 2000) and the case where only neighboring spikes are taken into account (Nearest-Spike interaction) (van Rossum et al., 2000; Bi, 2002; Izhikevich and Desai, 2003; Burkitt et al., 2004; Pfister and Gerstner, 2006). We found a slight preference for All-to-All interactions.

\section{Materials and Methods}

We compared a new triplet-based model with experimental data from the hippocampus and visual cortex. The visual cortex data set (Sjöström 
et al., 2001) used in this study consists of a standard pairing protocol in which the frequency of the pairing has been changed. We also considered a hippocampal culture data set (Wang et al., 2005) which consists of pair, triplet, and quadruplet protocols. Because both data sets disagree on some specific protocols (at low frequency of the pairing protocol, no potentiation is elicited in Sjöström's data, whereas a large amount of potentiation is present in Wang's data) and because the preparations are different, we fitted our models with different parameters for each data set.

Synaptic learning rule. Our new triplet-based model of STDP is an extension of classical pairbased STDP models. Traditional mechanistic models of STDP involve a small number of variables that are updated by presynaptic and postsynaptic firing events (Kistler and van Hemmen, 2000; Abarbanel et al., 2002; Gerstner and Kistler, 2002; Karmarkar and Buonomano, 2002). The new triplet rule is formulated in such a framework.

To introduce the variables used in our model, we considered the process of synaptic transmission. Whenever a presynaptic spike arrives at an excitatory synapse, glutamate is released into the synaptic cleft and binds to glutamate receptors. Let $r_{1}$ denote the amount of glutamate bound to a postsynaptic receptor. The variable $r_{1}$ increases whenever there is a presynaptic spike and decreases back to zero otherwise with a time constant of $\tau_{+}$. This can be written as follows:

$$
\begin{gathered}
\frac{d r_{1}(t)}{d t}=-\frac{r_{1}(t)}{\tau_{+}} \\
\text {if } t=t^{\text {pre }} \text {, then } r_{1} \rightarrow r_{1}+1 .
\end{gathered}
$$

Here, $t^{\text {pre }}$ denotes the moment of spike arrival at the presynaptic terminal. The units of $r_{1}$ are chosen such that glutamate binding increases by one unit after spike arrival. We emphasize that $r_{1}$ is an abstract variable. Instead of glutamate binding, it could describe equally well some other quantity that increases after presynaptic spike arrival. We call $r_{1}$ a detector of presynaptic events.

Instead of having only one process triggered by a presynaptic spike, it is possible to consider several different quantities, which increase in the presence of a presynaptic spike. In our model, we considered two different detectors of presynaptic events, namely $r_{1}$ and $r_{2}$. The dynamics of $r_{2}$ is analogous to that of $r_{1}$ except that its time constant $\tau_{x}$ is larger than $\tau_{+}$. Similarly, we assume that each postsynaptic spike $t^{\text {post }}$ induces an increase of two different quantities that we denote $o_{1}$ and $o_{2}$. Potential interpretations of $o_{1}$ and $o_{2}$ are given below. In the absence of postsynaptic spiking, these postsynaptic detectors decrease their value with a time constant $\tau_{-}$and $\tau_{y}$, respectively. Formally, this gives the following:

$$
\begin{aligned}
& \frac{d r_{2}(t)}{d t}=-\frac{r_{2}(t)}{\tau_{\mathrm{x}}} \quad \text { if } t=t^{\text {pre }} \text { then } r_{2} \rightarrow r_{2}+1 \\
& \frac{d o_{1}(t)}{d t}=-\frac{o_{1}(t)}{\tau_{-}} \quad \text { if } t=t^{\text {post }} \text { then } o_{1} \rightarrow o_{1}+1 \\
& \frac{d o_{2}(t)}{d t}=-\frac{o_{2}(t)}{\tau_{\mathrm{y}}} \quad \text { if } t=t^{\text {post }} \text { then } o_{2} \rightarrow o_{2}+1 .
\end{aligned}
$$

We do not want to identify the variables $r_{1}, r_{2}, o_{1}$, and $o_{2}$ with specific biophysical quantities. Candidates of detectors of presynaptic events are, for example, the amount of glutamate bound (Karmarkar and Buonomano, 2002) or the number of NMDA receptors in an activated state (Senn et al., 2001). Postsynaptic detectors $o_{1}$ and $o_{2}$ could represent the influx of calcium concentration through voltage-gated $\mathrm{Ca}^{2+}$ channels and NMDA channels (Karmarkar and Buonomano, 2002) or the number of secondary messengers in a deactivated state of the NMDA receptor (Senn et al., 2001) or the voltage trace of a back-propagating action potential (Shouval et al., 2002).

Because our present model is formulated as a mechanistic model, it is possible to define changes of synaptic efficacies for our triplet learning rule with All-to-All interactions as a function of those four detectors without making any assumption on the biophysical quantities they represent. We assume that the weight decreases after presynaptic spike arrival by an amount that is proportional to the value of the postsynaptic variable $o_{1}$ but depends also on the value of the second presynaptic detector $r_{2}$. Hence, presynaptic spike arrival at time $t^{\text {pre }}$ triggers a change given by the following:

$$
w(t) \rightarrow w(t)-o_{1}(t)\left[A_{2}^{-}+A_{3}^{-} r_{2}(t-\epsilon)\right] \text { if } t=t^{\mathrm{pre}} .
$$

Similarly, a postsynaptic spike at time $t^{\text {post }}$ triggers a change that depends on the presynaptic variable $r_{1}$ and the second postsynaptic variable $o_{2}$ as follows:

$$
w(t) \rightarrow w(t)+r_{1}(t)\left[A_{2}^{+}+A_{3}^{+} o_{2}(t-\epsilon)\right] \text { if } t=t^{\text {post }} .
$$

Here, $A_{2}^{+}$and $A_{2}^{-}$denote the amplitude of the weight change whenever there is a pre-post pair or a post-pre pair. Similarly, $A_{3}^{+}$and $A_{3}^{-}$denote the amplitude of the triplet term for potentiation and depression, respectively (Fig. 1A). All of the four amplitude parameters are assumed to be greater or equal to zero. $\varepsilon$ is a small positive constant to ensure that the weight is updated before the detectors $r_{2}$ or $o_{2}$. In other words, $r_{2}$ is zero unless a previous presynaptic spike has led to an increase of $r_{2}$. This ensures the detection of spike triplets.

Figure $1 B$ illustrates how a 1-pre-2-post triplet is detected by the learning rule. At the time of a postsynaptic spike, the learning rule "reads" the value of the second postsynaptic variable $o_{2}$ just before the spike (see the red dot at time $t^{\text {post }}-\varepsilon$ in Fig. $1 B$ ) as well as the value of the presynaptic 
Table 1. Experimental weight change $\Delta w$ as a function of the delay $\Delta t=t^{\text {post }}-$ $t^{\text {pre }}$ induced by a pairing protocol in the visual cortex

\begin{tabular}{lcc}
\hline & $\Delta t=10 \mathrm{~ms}$ & $\Delta t=-10 \mathrm{~ms}$ \\
\hline$\rho=0.1 \mathrm{~Hz}$ & $-0.04 \pm 0.05$ & $-0.29 \pm 0.08$ \\
$\rho=10 \mathrm{~Hz}$ & $0.14 \pm 0.1$ & $-0.41 \pm 0.11$ \\
$\rho=20 \mathrm{~Hz}$ & $0.29 \pm 0.14$ & $-0.34 \pm 0.1$ \\
$\rho=40 \mathrm{~Hz}$ & $0.53 \pm 0.11$ & $0.56 \pm 0.32$ \\
$\rho=50 \mathrm{~Hz}$ & $0.56 \pm 0.26$ & $0.75 \pm 0.19$
\end{tabular}

Those values are used for the fitting of the pair-based and triplet-based models of visual cortical neurons. Data were obtained from Sjöström (2001).

Table 2. Experimental weight change $\Delta w$ as a function of the relative spike timing $\Delta t, \Delta t_{1}, \Delta t_{2}$, and $T$ induced by pairing, triplet and quadruplet protocols in hippocampal cultures

The data are used for the fitting of the pair-based and triplet-based models of hippocampal culture neurons. Data were obtained from Wang et al. (2005).

\begin{tabular}{|c|c|c|c|c|c|}
\hline \multicolumn{2}{|l|}{ Pairing } & & \multicolumn{3}{|l|}{ Quadruplet } \\
\hline$\Delta w$ & $\overline{\Delta t(\mathrm{~ms})}$ & & $\Delta w$ & $T(\mathrm{~ms})$ & $\Delta t$ (ms) \\
\hline \multirow{3}{*}{$\begin{array}{r}0.25 \pm 0.05 \\
-0.17 \pm 0.05\end{array}$} & \multirow{3}{*}{$\begin{array}{r}10 \\
-10\end{array}$} & & \multirow{3}{*}{$\begin{array}{r}-0.003 \pm 0.03 \\
0.06 \pm 0.04 \\
0.21 \pm 0.04\end{array}$} & -88.5 & 5 \\
\hline & & & & 83.7 & 5 \\
\hline & & & & 20 & 5 \\
\hline \multicolumn{3}{|c|}{ Triplet (2-pre-1-post) } & \multicolumn{2}{|c|}{ Triplet (1-pre-2-post) } & \\
\hline$\Delta w$ & $\Delta t_{1}$ (ms) & $\Delta t_{2}$ (ms) & $\Delta w$ & $\Delta t_{1}$ (ms) & $\Delta t_{2}(\mathrm{~ms})$ \\
\hline$-0.01 \pm 0.04$ & 5 & -5 & $0.33 \pm 0.04$ & -5 & 5 \\
\hline $0.03 \pm 0.04$ & 10 & -10 & $0.34 \pm 0.04$ & -10 & 10 \\
\hline $0.01 \pm 0.03$ & 15 & -5 & $0.22 \pm 0.08$ & -5 & 15 \\
\hline $0.24 \pm 0.06$ & 5 & -15 & $0.29 \pm 0.05$ & -15 & 5 \\
\hline
\end{tabular}

$1 / \rho$. The interest of the study of Sjöström et al. (2001) is that the authors analyzed, in this pairing protocol, the weight change as a function of the frequency $\rho$ for a fixed $\Delta t$. Changing the frequency $\rho$ is a good way to check the validity of a model, especially at high frequency, where many spikes are potentially in the temporal range of interaction.

It should be noted that the amount of potentiation for a pre-post $(\Delta t=$ $10 \mathrm{~ms}$ ) pair reported by Wang et al. (2005) is significantly lower than the one originally measured (Bi and Poo, 1998) under the same conditions. As mentioned by Wang et al. (2005), this can be accounted for by the difference in initial synaptic strength, which was higher in the study by $\mathrm{Bi}$ and Poo (1998). To test our model on a consistent set of data, we took the measurements of Wang et al. (2005) (compare their supplemental Fig. 1) (i.e., $\Delta w \simeq 0.25 \pm 0.05$ for $\Delta t=+10 \mathrm{~ms}$ and $\Delta w \simeq-0.17 \pm 0.05$ for $\Delta t=-10 \mathrm{~ms}$. Data from Wang et al. (2005), including error bars, are redrawn in Figure 3. Because the potentiation and depression time constant are not present in the study by Wang et al. (2005), we took $\tau_{+}=16.8 \mathrm{~ms}$ and $\tau_{-}=33.7$ from (Bi and Poo, 2001).

Triplet protocol. The first triplet protocol (see Fig. 2C) consists of $n=60$ sets of three spikes repeated at a given frequency $\rho=1 \mathrm{~Hz}$. Each triplet consists of two presynaptic spikes and one postsynaptic spike characterized by $\Delta t_{1}=$ $t^{\text {post }}-t_{1}^{\text {pre }}$ and $\Delta t_{2}=t^{\text {post }}-t_{2}^{\text {pre }}$ where $t_{1}^{\text {pre }}$ and $t_{2}^{\text {pre }}$ are the first and second presynaptic spikes of the triplet.

The second triplet protocol (see Fig. $2 D$ ) also detector $r_{1}$ (see the blue dot at time $t^{\text {post }}$ in Fig. $1 B$ ) and increases the weight by an amount $A_{2}^{+} r_{1}\left(t^{\text {post }}\right)+A_{3}^{+} r_{1}\left(t^{\text {post }}\right) o_{2}\left(t^{\text {post }}-\varepsilon\right)($ see Eq. 4$)$.

Note that if we set $A_{3}^{+}=0$ and $A_{3}^{-}=0$, the model becomes a classical pair-based STDP model (Gerstner et al., 1996; Kempter et al., 1999; Kistler and van Hemmen, 2000; Song et al., 2000). This pair-based STDP model was used for the results of Figure 2. It should be further noted that the two extra triplet terms vanish if a single spike pair is presented or if spike pairings are repeated at low frequency. This means that in the limit of low frequency, the classical pair-based learning rule is identical to our triplet learning rule.

The triplet learning rule of Equations 3 and 4 can also describe a Nearest-Spike interaction scheme if we redefine the update rule of presynaptic and postsynaptic detectors. Instead of simply low-pass filtering the spike trains (i.e., adding the effects of all spikes), the detector variables saturate at 1 (i.e., $0 \leq r_{1}, r_{2}, o_{1}, o_{2} \leq 1$ ). This is achieved by updating the variables to the value of 1 instead of updating by at step of 1 . In this way, the synapse forgets all other previous spikes and keeps only the memory of the last one (Fig. 1C).

In this paper, we consider first a full triplet model, which takes into account all four terms of Equations 3 and 4 . Then, we will see that only some of the terms are really necessary. This is why we define two different minimal models. The first one is intended to fit the visual cortex data and disregards two terms (i.e., $A_{2}^{+}=0$ and $A_{3}^{-}=0$ ). For the hippocampal culture data set, we consider a slightly different minimal model, which disregards only one term (i.e., $A_{3}^{-}=0$ ).

In principle, the amplitude parameters $A_{2}^{+}, A_{2}^{-}, A_{3}^{+}$, and $A_{3}^{-}$could change on a slow time scale. For example, similar to the threshold in the BCM rule, those parameters could, because of homeostatic processes, depend on the mean postsynaptic firing rate $\rho_{y}$ averaged over a time scale of $10 \mathrm{~min}$ or more.

Protocols. To compare our model to experimental data, we followed three different experimental protocols (see Fig. 2) in which the synaptic weight changes as a function of the presynaptic and postsynaptic spike statistics. The forth protocol is of a more theoretical value in the sense that it can be compared with the BCM learning rule, which has interesting computational properties.

Pairing protocol. This is the classical STDP protocol (see Fig. 2A) (Markram et al., 1997; Bi and Poo, 1998, 2001; Zhang et al., 1998; Sjöström et al., 2001; Froemke and Dan, 2002). $n=60$ pairs of presynaptic and postsynaptic spikes shifted by $\Delta t$ are elicited at regular intervals of consists of $n=60$ triplets. The only difference is that each triplet consists of one presynaptic and two postsynaptic spikes. In this case, $\Delta t_{1}=$ $t_{1}^{\text {post }}-t^{\text {pre }}$ and $\Delta t_{2}=t_{2}^{\text {post }}-t^{\text {pre }}$, where $t_{1}^{\text {post }}$ and $t_{2}^{\text {post }}$ are, respectively, the first and second postsynaptic spikes of the triplet.

Experiments with such a triplet protocol have been performed by Froemke and Dan (2002) in L2/3 pyramidal neurons of the rat visual cortex and by Wang et al. (2005) in hippocampal cultures. To have a consistent and broad data set (i.e., pair, triplet, and quadruplet experiments), we decided, in the present study, to focus only on the data of Wang et al. (2005), because we did not find enough quantitative information about quadruplets in the study by Froemke and Dan (2002).

Quadruplet protocol. This protocol consists of $n=60$ quadruplets at frequency $\rho=1 \mathrm{~Hz}$ (see Fig. 2B). It was used by Wang et al. (2005) and is characterized as follows: a post-pre pair with a delay of $\Delta t_{1}=t_{1}^{\text {post }}-$ $t_{1}^{\text {pre }}<0$ is followed after a time $T$ by a pre-post pair with a delay of $\Delta t_{2}=$ $t_{2}^{\text {post }}-t_{2}^{\text {pre }}>0$. When $T$ is negative, the opposite happens. A pre-post pair $\left(\Delta t_{2}=t_{2}^{\text {post }}-t_{2}^{\text {pre }}>0\right)$ is followed by a post-pre pair $\left(\Delta t_{1}=t_{1}^{\text {post }}-\right.$ $\left.t_{1}^{\text {pre }}<0\right)$. Formally, $T$ is defined by $T=\left(t_{2}^{\text {pre }}+t_{2}^{\text {post }}\right) / 2-\left(t_{1}^{\text {pre }}+\right.$ $\left.t_{1}^{\text {post }}\right) / 2$. Throughout this paper, we took $\Delta t=-\Delta t_{1}=\Delta t_{2}=5 \mathrm{~ms}$.

Poisson protocol. The presynaptic and postsynaptic spike trains are Poisson spike trains with firing rate $\rho_{x}$ and $\rho_{y}$, respectively. The interest of such a protocol is that it is possible to establish a link with the BCM learning rule (Bienenstock et al., 1982), which has attractive theoretical properties. Indeed, this learning rule was originally used to explain the emergence of orientation selectivity in the visual cortex. Even if this protocol has less experimental support than the other protocols, some aspects of it have been indirectly measured in the visual cortex (Kirkwood et al., 1996) and in hippocampal slice (Artola et al., 1990; Dudek and Bear, 1992).

Data fitting. To fit the amplitude parameters $A_{2}^{+}, A_{2}^{-}, A_{3}^{+}$, and $A_{3}^{-}$and the time constants $\tau_{x}$ and $\tau_{y}\left(\tau_{+}=16.8 \mathrm{~ms}\right.$ and $\tau_{-}=33.7 \mathrm{~ms}$ are kept fixed), we calculated the total weight change $\Delta w_{i}^{\bmod }$ for a given pairing or triplet protocol and compared it to the experimental value $\Delta w_{i}^{\exp }$. For the optimization of the parameters, we performed a minimization of the normalized mean-square error $E$ defined by the following;

$$
E=\frac{1}{P} \sum_{i=1}^{P}\left(\frac{\Delta w_{\mathrm{i}}^{\exp }-\Delta w_{\mathrm{i}}^{\bmod }}{\sigma_{i}}\right)^{2}
$$


where $\Delta w_{i}^{\exp }$ and $\sigma_{i}$ are the experimental mean weight change and SEM weight change for a given data point i. $P$ is the number of data points within a data set; $p=10$ for the visual cortex data set (Table 1 ), and $p=$ 13 for the hippocampal culture data set (Table 2). $\Delta w_{i}^{\text {mod }}$ is the weight change for a given model (pair or triplet model).

Numerical procedures. The weight change $\Delta w^{\text {mod }}$ for a given model and a given protocol can be either simulated numerically with Equations 1-4 or calculated analytically. See supplemental material (available at www.jneurosci.org) for an example of analytical calculation of the weight change of the triplet model applied to the pairing protocol with NearestSpike interactions.

In the present study, the weight changes predicted by all different models (pair-based models, minimal and full triplet-based models with both Nearest-Spike and All-to-All interactions) have been calculated analytically and then evaluated numerically with MatLab (MathWorks, Natick, MA) on a Sun machine. The normalized mean-square error $E$ of Equation 5 has been minimized with the MatLab built-in function Isqnonlin, which uses a reflective Newton method.

\section{Results}

\section{Standard pair-based STDP models fail to reproduce} frequency effects

In a first series of experiments, we applied a classical pair-based STDP learning rule (compare Eqs. 3 and 4 with $A_{3}^{-}=0$ and $A_{3}^{+}=$ $0)$ to the pairing protocol with 60 pairs of presynaptic and postsynaptic spikes (see Materials and Methods). Obviously, the weight change predicted by the model depends on the precise choice of the parameters $A_{2}^{+}, A_{2}^{-}, \tau_{+}$, and $\tau_{-}$. We therefore set those parameters in such a way that the normalized mean square error $E$ across all experimental protocols is minimal (see Eq. 5). We found that even with the best set of parameters, the classical STDP model fails, for both the All-to-All interaction and the Nearest-Spike interaction, to reproduce the experimental data (Fig. 2A). This is attributable to the following reasons.

First, as pointed out by Sjöström et al. (2001), a surprising aspect of their finding is that at low repetition frequency, $\rho$, there is no potentiation. This cannot be captured by standard pairbased STDP models, because for any choice of the parameter $A_{2}^{+}>0$, the pair-based model induces LTP if a presynaptic spike precedes a postsynaptic one by a few milliseconds.

Second, as we can see in Figure 2, for $\Delta t>0$, potentiation increases when frequency increases. This behavior can also not be reproduced by classical STDP models. Indeed, in pair-based STDP models, as soon as the frequency increases, the pre-post pairs approach each other and generate an interaction between the postsynaptic spike of one pair and the presynaptic spike of the next pair. The effect of these post-pre pairs should increase with frequency and therefore depress the synapse, which is not what is seen in the experiments. Therefore, classical pair-based models fail to reproduce the pairing experiment of Sjöström et al. (2001).

It should be noted that the absence of potentiation at low frequency is in direct conflict with the results of $\mathrm{Bi}$ and $\mathrm{Poo}$ (1998), Zhang et al. (1998), and Froemke and Dan (2002), where there is a reasonable potentiation at low frequency. Because the preparation of Sjöström et al. (2001) is different from the one of Bi and Poo (1998) and Wang et al. (2005) and the results in conflict, it seems natural to use different parameters in our model for each data set.

\section{Standard pair-based STDP models fail to reproduce triplet and quadruplet experiments}

The following is a second set of evidence of the limits of pairbased STDP learning rules. In triplet experiments (Fig. 2C,D), there is a clear asymmetry between a pre-post-pre and a post-pre-
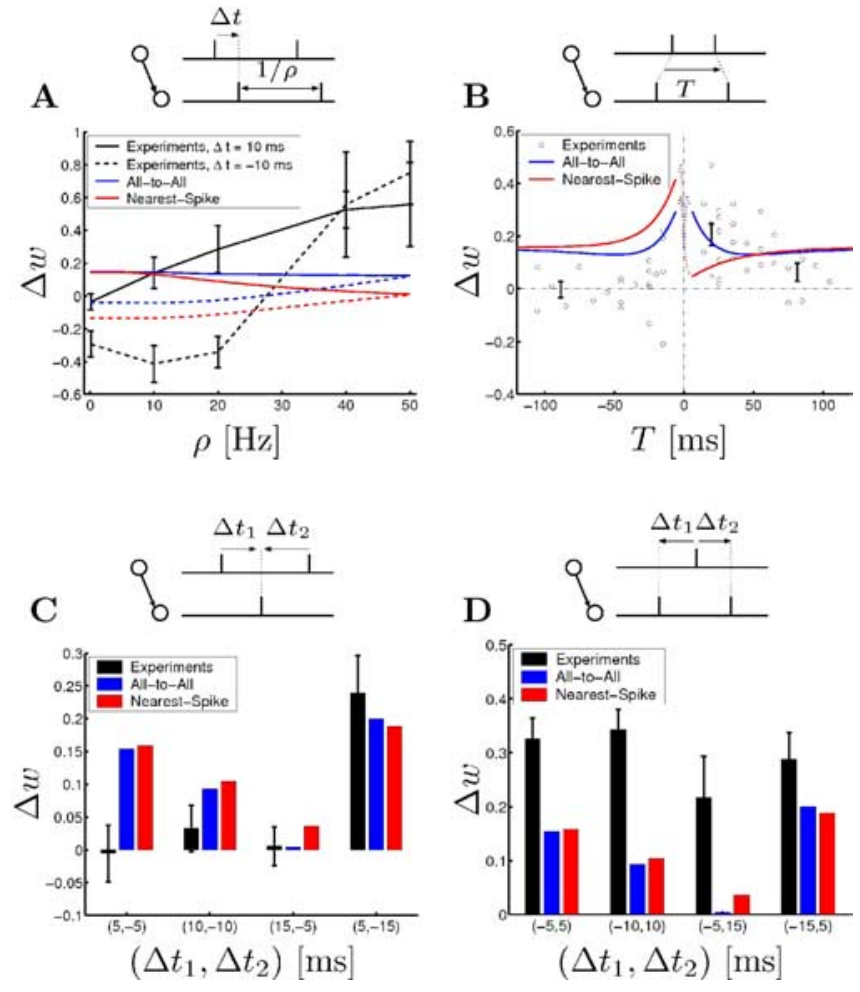

Figure 2. Failure of pair-based STDP learning rules. In all four subgraphs, black lines or symbols denote experimental data, blue lines correspond to the All-to-All pair model, and the red lines correspond to the Nearest-Spike pair model (see Results for details). $\boldsymbol{A}$, Weight change in a pairing protocol as a function of the frequency $\rho$ (solid lines, $\Delta t=+10 \mathrm{~ms}$; dashed lines, $\Delta t=-10 \mathrm{~ms}$ ). Black lines and data points (with errors) are redrawn from Sjöström et al. (2001). The experimental data are reproduced at neither high nor low values of the repetition frequency $\rho$. B, Quadruplet protocol. Black circles are redrawn from Wang et al. (2005). C, D, Triplet protocol for the pre-post-pre case $(\boldsymbol{C})$ and the post-pre-post case $(\boldsymbol{D})$. The black dots in $\boldsymbol{B}$ and the black bars (and SEs) in $\boldsymbol{C}$ and $\boldsymbol{D}$ are redrawn from Wang et al. (2005). The asymmetry of the experimental results [no potentiation for $\left(\Delta t_{1}, \Delta t_{2}\right)=(5 \mathrm{~ms},-5 \mathrm{~ms})$ ] in $C$ but strong potentiation for ( $-5 \mathrm{~ms}, 5 \mathrm{~ms}$ ) in $\boldsymbol{D}$ is not captured by the pair-based models.

post experiment. For example, 60 repetitions of a pre-post-pre triplet with relative timing $\left(\Delta t_{1}, \Delta t_{2}\right)=(5 \mathrm{~ms},-5 \mathrm{~ms})$ yields no weight change, whereas the same number of repetitions of a postpre-post triplet with $\left(\Delta t_{1}, \Delta t_{2}\right)=(-5 \mathrm{~ms}, 5 \mathrm{~ms})$ yields a weight change of $\sim 30 \%$. However, any pair-based model would predict the same result for pre-post-pre and post-pre-post experiments, because the same pairs occur. Therefore, triplet results cannot be explained by a sum of a pre-post potentiation term and a post-pre depression term (Fig. 2C,D).

Finally, the asymmetry present in the quadruplets experiments (Fig. $2 B$ ) also causes some problems for pair-based STDP models. A quadruplet consists of a pre-post-post-pre sequence or a post-pre-pre-post sequence, and $|T|$ denotes the interval between the first and last pair of spikes within the quadruplet (see Materials and Methods for more details). In a pair-based model with All-to-All interactions and for a given interval $|T|$ between the pairs, the weight changes for post-pre-pre-post and pre-postpost-pre are strictly identical because of the symmetry of the protocol and the symmetry of the All-to-All interaction. The weight change predicted by a pair model can therefore not explain the asymmetry seen in the data. With Nearest-Spike interactions, the situation gets even worse: pre-post-post-pre quadruplets consist of two pre-post pairs and one post-pre term, whereas for the post-pre-pre-post case, the opposite occurs: two post-pre pairs and only one pre-post pair. Therefore, the Nearest-Spike 
Table 3. Visual cortex data set

\begin{tabular}{|c|c|c|c|c|c|c|c|c|}
\hline Model & & $A_{2}^{+}$ & $A_{3}^{+}$ & $A_{2}^{-}$ & $A_{3}^{-}$ & $\tau_{\mathrm{x}}(\mathrm{ms})$ & $\tau_{y}(\mathrm{~ms})$ & $E$ \\
\hline \multirow[t]{2}{*}{ All-to-All } & Full & $5 \times 10^{-10}$ & $6.2 \times 10^{-3}$ & $7 \times 10^{-3}$ & $2.3 \times 10^{-4}$ & (101) & 125 & 0.33 \\
\hline & Min. & 0 & $6.5 \times 10^{-3}$ & $7.1 \times 10^{-3}$ & 0 & & 114 & 0.34 \\
\hline \multirow[t]{2}{*}{ Nearest-Spike } & Full & $8.8 \times 10^{-11}$ & $5.3 \times 10^{-2}$ & $6.6 \times 10^{-3}$ & $3.1 \times 10^{-3}$ & 714 & 40 & 0.22 \\
\hline & Min. & 0 & $5 \times 10^{-2}$ & $8 \times 10^{-3}$ & 0 & & 40 & 0.34 \\
\hline
\end{tabular}

Table 4. Hippocampal culture data set

\begin{tabular}{|c|c|c|c|c|c|c|c|c|}
\hline Model & & $A_{2}^{+}$ & $A_{3}^{+}$ & $A_{2}^{-}$ & $A_{3}^{-}$ & $\tau_{\mathrm{x}}(\mathrm{ms})$ & $\tau_{y}$ (ms) & $E$ \\
\hline \multirow[t]{2}{*}{ All-to-All } & Full & $6.1 \times 10^{-3}$ & $6.7 \times 10^{-3}$ & $1.6 \times 10^{-3}$ & $1.4 \times 10^{-3}$ & 946 & 27 & 2.9 \\
\hline & Min. & $5.3 \times 10^{-3}$ & $8 \times 10^{-3}$ & $3.5 \times 10^{-3}$ & 0 & & 40 & 3.4 \\
\hline \multirow[t]{2}{*}{ Nearest-Spike } & Full & $4.6 \times 10^{-3}$ & $9.1 \times 10^{-3}$ & $3 \times 10^{-3}$ & $7.5 \times 10^{-9}$ & (575) & 47 & 2.9 \\
\hline & Min. & $4.6 \times 10^{-3}$ & $9.1 \times 10^{-3}$ & $3 \times 10^{-3}$ & 0 & & 48 & 2.9 \\
\hline
\end{tabular}

List of parameters used to model the hippocampal culture data set. In this table, the terms "full" and "min." represent full triplet model and minimal triplet model, respectively. The additional parameters $\tau_{+}=16.8 \mathrm{~ms}$ and $\tau_{-}=33.7 \mathrm{~ms}$ are taken from Bi and Poo (2001) and kept fixed for all models and data sets. In some cases, parentheses are added in the $\tau_{\mathrm{x}}$ column to indicate that the error function is insensitive to the exact value of $\tau_{\mathrm{x}}$ in those cases. The last column corresponds to the fitting error given by Equation 5 and plotted in Figure 6.

interaction scheme leads to an asymmetry that is opposite to the one found in experiments (Fig. 2B).

\section{Triplet rule}

So far, we have shown that standard pair-based STDP models fail to reproduce frequency effects of the pairing protocol as well as triplet and quadruplet experiments. This is mainly because of the fact that pair-based models are intrinsically symmetric, in the sense that they predict the same weight change for a pre-post pair followed by a post-pre pair with the same delay $\Delta t$ as for the inverted order [i.e., a post-pre pair followed by a pre-post pair (with the same delay $\Delta t$ )]. However, there is no a priori reason to think that a pre-post-pre and a post-pre-post triplet should give the same result because they will activate different presynaptic and postsynaptic pathways.

We therefore included extra terms in the learning rule to break the symmetry induced by pair-based models. Specifically, we added a triplet depression term (i.e., a 2-pre-1-post term) as well as a triplet potentiation term (i.e., a 1-pre-2-post term) (see Materials and Methods for more details). We call this model a full triplet model, because it includes both pair terms and triplet terms. The full triplet model is described by eight parameters: four amplitude parameters $A_{2}^{-}, A_{2}^{+}, A_{3}^{-}$, and $A_{3}^{+}$and four time constants $\tau_{+}, \tau_{-}, \tau_{x}$, and $\tau_{y}$. Note that pair-based models are described by four parameters $\left(A_{2}^{-}, A_{2}^{+}, \tau_{+}\right.$, and $\left.\tau_{-}\right)$.

In analogy to our approach in the previous subsection, we applied our triplet model to the protocols described in Materials
A

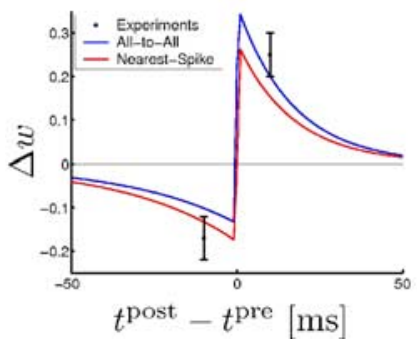

B

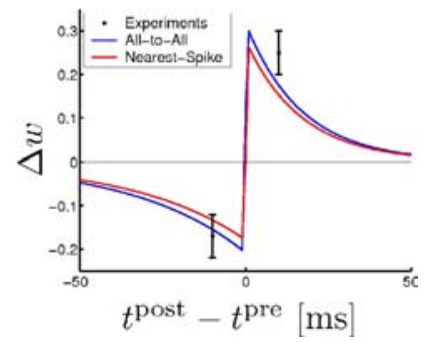

Figure 3. The triplet learning rule can reproduce the STDP learning window. Weight change induced by a repetition of 60 pairs of presynaptic and postsynaptic spike with a delay of $\Delta t$ at a repetition frequency of $1 \mathrm{~Hz}$. $\boldsymbol{A}$, Weight change as a function of the time difference between postsynaptic and presynaptic spike timing for the full triplet model $(\boldsymbol{A})$ and the minimal triplet model $(\boldsymbol{B})$. The parameters taken for the triplet models are those that correspond to the hippocampal culture data (Tables 3, 4). Experimental data points and SEs are redrawn from Wang et al. (2005). and Methods. More precisely, we calculated analytically for each protocol the weight change predicted by our triplet learning rule (see supplemental material, available at www.jneurosci.org, for an example of explicit expression of the weight change). As before, we want our triplet learning rule to fit as best as possible to the experimental data of Sjöström et al. (2001) or Wang et al. (2005). We therefore minimized the normalized mean square error across all data points of a given data set (Table 1 or 2 ) by adjusting the eight parameters mentioned above. The resulting parameters are summarized in Tables 3 and 4.

As a first test for the triplet learning rule, we checked whether it can reproduce the biphasic learning window observed by $\mathrm{Bi}$ and Poo (1998). Our triplet learning rule succeeds to reproduce the classical STDP learning window (Fig. 3), because the triplet terms specific to our model play a minor role at a fixed low frequency.

\section{Triplet learning rules can reproduce frequency effects}

In this section, we study the pairing protocol used by Sjöström et al. (2001) in visual cortex (i.e., we apply 60 pairs of presynaptic and postsynaptic spikes at a given frequency $\rho$ ). As shown in Figure $4 A$, our full triplet learning rule succeeds to reproduce frequency effects of the pairing protocol. Indeed, the two main problems of the pair-based STDP models explained in section three for the pairing protocol are solved by the triplet model for the following reasons. First, the absence of potentiation at low frequency is achieved by setting $A_{2}^{+}$to a low value; second, the increase of potentiation with frequency is implemented via the triplet potentiation term controlled by $A_{3}^{+}$, which has a stronger effect than the triplet depression term $A_{3}^{-}$(Table 3 ). Thus, our model can explain results at different frequencies without an explicit "potentiation wins" mechanism suggested previously (Sjöström et al., 2001; Wang et al., 2005).

Because some of the optimized parameters of the triplet learning rule have values close to zero, we concluded that the terms controlled by these parameters can be neglected. This allowed us to define a minimal triplet model with less parameters. The first parameter we can easily drop is the amplitude $A_{2}^{+}$of the pair potentiation term, because it is extremely small in both the Allto-All and Nearest-Spike interaction scheme (Table 3). The second parameter we neglect is $A_{3}^{-}$. This is possible for the following reason. In the All-to-All interaction scheme, we have $A_{3}^{-} \ll A_{2}^{-}$. Therefore, the effect of the triplet depression term is negligible compared with the depression induced by spike pairs.

Results with the minimal triplet model show good agreement with experimental data (Fig. 5A). Hence, the minimal model with five parameters can explain the visual cortex data that the classical pair-based STDP model with four parameters fails to explain.

\section{Triplet learning rules can reproduce triplet and quadruplet experiments}

By following the same procedure as the one described in the previous paragraph, we applied our full triplet learning rule to the second set of data (i.e., the hippocampal culture data set) (Bi and Poo, 1998, 2001; Wang et al., 2005). The parameters resulting from the minimization of the normalized mean square error 

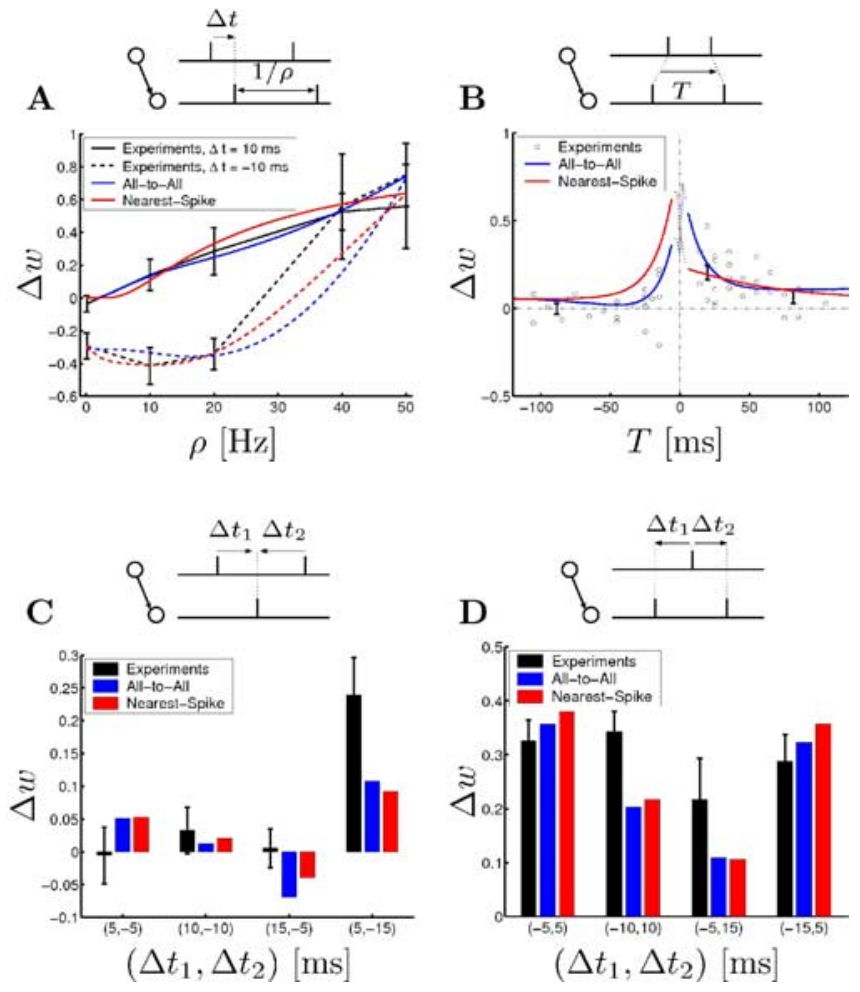

Figure 4. The full triplet learning rule succeeds to reproduce the pairing experiment and most of the triplet and quadruplet experiments. In all four subgraphs, the black lines and circles denote experimental data, the blue lines correspond to the All-to-All pair model, and the red lines correspond to the Nearest-Spike pair model. $\boldsymbol{A}$, Weight change in a pairing protocol as a function of the frequency $\rho$ (solid lines, $\Delta t=+10 \mathrm{~ms}$; dashed lines, $\Delta t=-10 \mathrm{~ms}$ ). The black lines and data points (with errors) are redrawn from Sjöström (2001). B, Quadruplet protocol. The black circles are redrawn from Wang et al. (2005). C, D, Triplet protocol for the pre-post-pre case $(\boldsymbol{C})$ and the post-pre-post case (D). The black dots in $\boldsymbol{B}$ and black bars (and SEs) in $\boldsymbol{C}$ and $\boldsymbol{D}$ are redrawn from Wang et al. (2005). The triplet-based models succeed to reproduce the asymmetry in triplets protocols [no potentiation for $\left(\Delta t_{1}, \Delta t_{2}\right)=(5 \mathrm{~ms},-5 \mathrm{~ms})$ in $C$ and strong potentiation for $(-5 \mathrm{~ms}, 5 \mathrm{~ms})]$ in $\boldsymbol{D}$ : for those triplets, the model results (with All-to-All interactions) are within $1.1 \sigma$ (SE of experimental data), whereas the results of the pair-based models are off by $>4 \sigma$.

across the pair, triplet, and quadruplet data are summarized in Table 3.

Our triplet learning rule does not only reproduce the classical STDP learning window (Fig. 3), but it also captures the results of most of the triplet and the quadruplet experiments. See Figure $4 B-D$. For example, the asymmetry between the pre-post-pre $\left[\left(\Delta t_{1}, \Delta t_{2}\right)=(5 \mathrm{~ms},-5 \mathrm{~ms})\right]$ and the post-pre-post $\left[\left(\Delta t_{1}, \Delta t_{2}\right)=\right.$ $(-5 \mathrm{~ms}, 5 \mathrm{~ms})]$ triplets can be well captured by our model. For those two specific triplet protocols, the predicted weight change of the full triplet learning rule with All-to-All interactions is within $1.1 \sigma(\mathrm{SEM})$ off the experimental mean weight change, whereas the pair-based learning predictions are off by $>4 \sigma$. We should, however, note that even if our triplet learning rule captures most of the triplet experiments, the fit is not perfect. For example, the pre-post-pre [with $\left(\Delta t_{1}, \Delta t_{2}\right)=(5 \mathrm{~ms},-15 \mathrm{~ms})$ ] triplet experiment is not well reproduced by our triplet learning rule (Figs. $4 C, 5 C$ ).

With arguments similar to those applied above to model the visual cortex data set, it is possible to reduce the complexity of the model of the hippocampal data set. Specifically, we have set $A_{3}^{-}=$ 0 as done previously. However, in contrast to the above minimal model for visual cortex data, the pair term controlled by $A_{2}^{+}$is kept as part of the model, because it is necessary to explain the
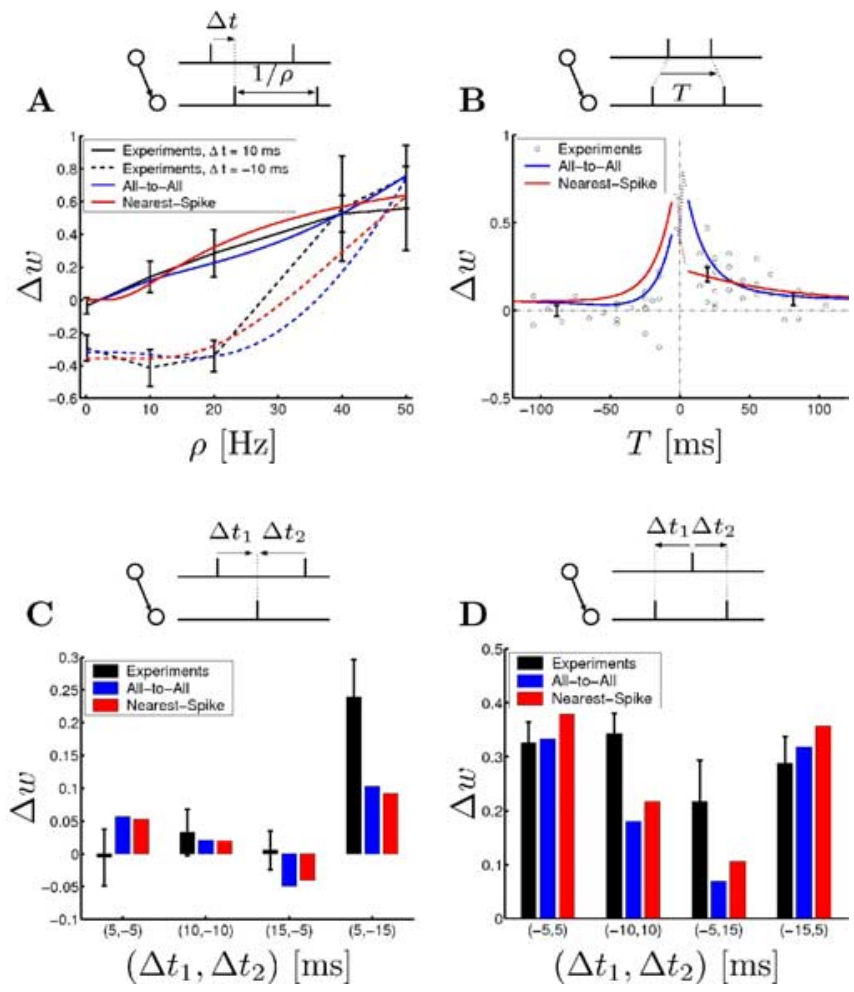

Figure 5. Minimal triplet learning rules are almost as good as full triplet learning rules. In all four subgraphs, the black line or circle denote experimental data, blue lines correspond to the All-to-All pair model, and the red lines correspond to the Nearest-Spike pair model. $\boldsymbol{A}$. Weight change in a pairing protocol as a function of the frequency $\rho$ (solid lines, $\Delta t=10 \mathrm{~ms}$; dashed lines, $\Delta t=-10 \mathrm{~ms}$ ). Black lines and data points (with errors) are redrawn from Sjöström (2001). B, Quadruplet protocol. Black circles are redrawn from Wang et al. (2005). C, D, Triplet protocol for the pre-post-pre case $(\boldsymbol{C})$ and the post-pre-post case $(\boldsymbol{D})$. Black dots in $\boldsymbol{B}$ and black bars (and SEs) in $\boldsymbol{C}$ and $\boldsymbol{D}$ are redrawn from Wang et al. (2005).

potentiation at $1 \mathrm{~Hz}$ repetition frequency. The resulting weight change of the minimal model applied to the triplet and quadruplet experiments is depicted in Figure $5 B-D$. We emphasize that the minimal model for the hippocampal data is different from the one used for the visual cortex data.

To compare the pair models and the minimal and full triplet models, we plotted the fitting error given by Equation 5 as a function of the number of parameters in the model (Fig. 6). The best types of model are those that can predict the experimental data as well as possible while being as simple as possible (i.e., having as few parameters as possible). In this sense, the minimal models are the best, because they perform almost as well as the full triplet models while having only one extra parameter compared with standard pair-based models (two extra parameters for the hippocampal culture data set).

Finally, for future tests of the triplet models, we propose two new protocols that have not yet been used experimentally. The first protocol consists of pre-post-pre triplets with relative timing $\left(\Delta t_{1}, \Delta t_{2}\right)=(5 \mathrm{~ms},-5 \mathrm{~ms})$, and the second protocol consists of post-pre-post triplets with relative timing $\left(\Delta t_{1}, \Delta t_{2}\right)=(-5 \mathrm{~ms}, 5$ ms). Triplets are repeated 60 times at different frequencies $\rho$. Figure $6, C$ and $D$, depicts the weight change predicted by the minimal triplet models (with All-to-All and Nearest-Spike interactions) for the two triplet protocols. The models predict a frequency dependence with a positive slope. However, the overall level of potentiation predicted by the All-to-All model is clearly different from that of the Nearest-Spike interaction model. Thus, 


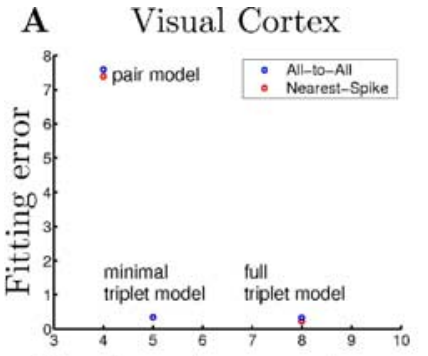

Number of parameters

C

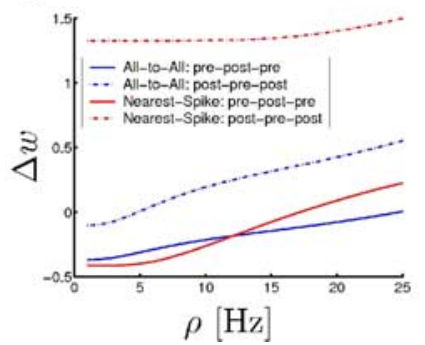

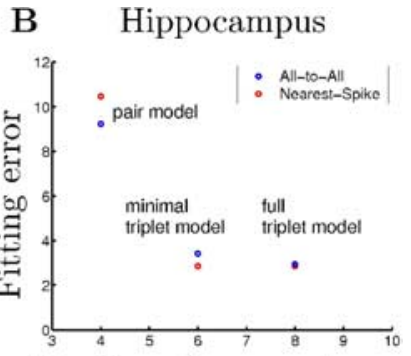

Number of parameters

D

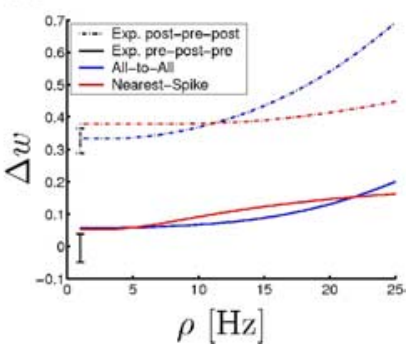

Figure 6. $\boldsymbol{A}, \boldsymbol{B}$, Comparison between the pair and triplet models. $\boldsymbol{C}, \boldsymbol{D}$, Predictions of the triplet models. $\boldsymbol{A}$, Fitting error (compare Eq. 5) for the visual cortex data set of Sjöström et al. (2001) as a function of the number of parameters in the model. The minimal model has only one extra parameter compared with a pair-based model but performs $>20$ times better. $\boldsymbol{B}$, Fitting error for the hippocampal data set of Wang et al. (2005). C, Predicted weight change (visual cortex) of the triplet protocol [solid lines, pre-post-pre with $\left(\Delta t_{1}, \Delta t_{2}\right)=(+5,-5) \mathrm{ms}$; dashed lines, post-pre-post with $\left.\left(\Delta t_{1}, \Delta t_{2}\right)=(-5,+5) \mathrm{ms}\right)$ with All-to-All interactions (blue lines) and with Nearest-Spike interactions (red lines)]. D, Same as in ( but for the hippocampal culture data set. Black bars correspond to the experimental results also present in subplots $C$ and D of Figures 2, 4, and 5.

the above experimental protocol would allow to test the triplet models and distinguish between its two variants.

Triplet learning rule can be mapped to the BCM learning rule Functional consequences of our new triplet model can be studied in two different ways (i.e., analytically or by numerical simulations). We used a combination of the two and proceeded as follows. First, we show analytically a close analogy ("mapping") between our triplet model and the traditional BCM theory. As a result of this mapping, we may conclude that, under random spike arrival with rate $\rho_{x}$, our triplet model behaves as a BCM model and inherits all of its functional properties. In particular, we expect that our triplet model exhibits synaptic competition leading to input selectivity as required for receptive field development. In a second step, we tested this prediction of input selectivity by numerical simulation.

First, we show that unlike standard pair-based STDP learning rules, our triplet learning rule can be mapped to the BCM learning rule. If we assume that the presynaptic and postsynaptic spike trains have Poisson statistics with $\rho_{x}$ and $\rho_{y}$, respectively, as firing rate, the expected weight change can be calculated analytically. Intuitively, we may expect that a triplet term with one presynaptic and two postsynaptic spikes leads to a weight change that is proportional to the postsynaptic rate and the square of the presynaptic rate. An analogous argument holds for the other terms. Indeed, a detailed calculation for the All-to-All triplet learning rule based on Equations 1-4 yields an expected weight change as follows:

A
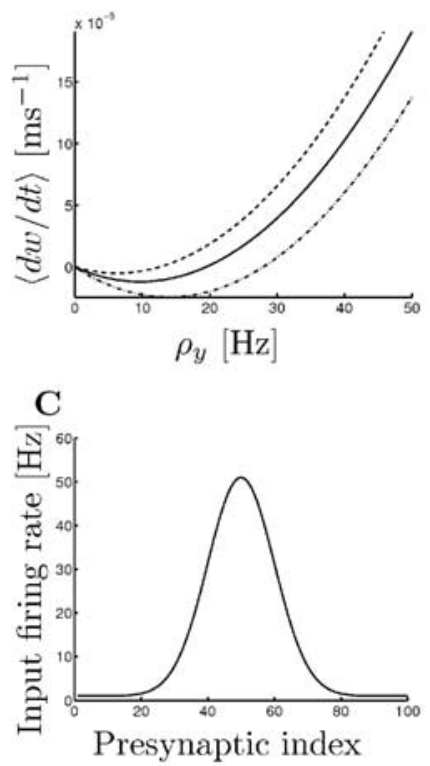

B

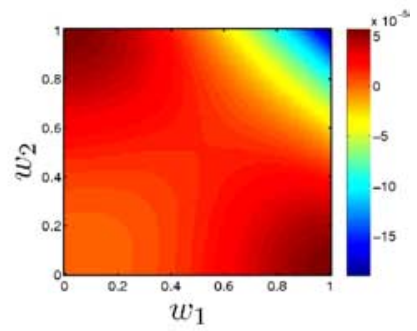

D

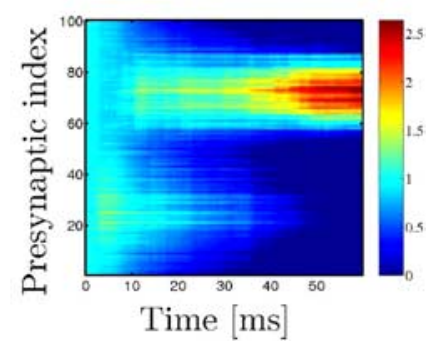

Figure 7. The triplet learning rule can be mapped to a BCM learning rule. $\boldsymbol{A}$, Instantaneous weight change as a function of the postsynaptic frequency for a minimal triplet model. (compare Eq. 6 with $A_{3}^{-}=0$ ). The presynaptic and postsynaptic spike trains are Poisson spike trains. The dashed line corresponds to $\lambda=\rho_{y}^{p} / \rho_{0}^{p}=0.64$, the solid line corresponds to $\lambda=1$, and the dashed line corresponds to $\lambda=1.44$. $B$, Energy landscape produced by the minimal triplet learning rule (with $p=2$ and $\rho_{0}=10 \mathrm{~Hz}$ ) in a two-input environment: $\rho_{x}^{1}=(10 \mathrm{~Hz}, 0)^{T}$ and $\rho_{x}^{2}=(0,10 \mathrm{~Hz})^{T}$. The presence of two specialized (and stable) fixed points as well as two unspecialized (and unstable) fixed points is an essential feature of the BCM learning rule. $\boldsymbol{C}$, Gaussian stimulation profile across 100 presynaptic neurons. The center of the Gaussian is shifted randomly every $200 \mathrm{~ms}$ to one of 10 random positions. Periodic boundary conditions are assumed. $\boldsymbol{D}$, Evolution of the 100 weights as a function of time under the stimulation described in $\boldsymbol{C}$. After 1 min of stimulation, the postsynaptic neuron becomes sensitive to a stimulation centered around the 70th presynaptic neuron. The parameters taken in the minimal model are those that correspond to the visual cortex (compare Tables 3 and 4).

$$
\left\langle\frac{d w}{d t}\right\rangle=-A_{2}^{-} \tau_{-} \rho_{\mathrm{x}} \rho_{\mathrm{y}}-A_{3}^{-} \tau_{-} \tau_{\mathrm{x}} \rho_{\mathrm{x}}^{2} \rho_{\mathrm{y}}+A_{2}^{+} \tau_{+} \rho_{\mathrm{x}} \rho_{\mathrm{y}}+A_{3}^{+} \tau_{+} \tau_{\mathrm{y}} \rho_{\mathrm{x}} \rho_{\mathrm{y}}^{2}
$$

Figure $7 A$ depicts the expected weight change of Equation 6 as a function of the postsynaptic frequency $\rho_{y}$. The above weight dynamics can be written as a BCM learning rule. Indeed, the BCM theory requires first that the weight change can be written as $d w / d t=\phi\left(\rho_{y}, \theta\right) \rho_{x}$, where $\phi$ is such that $\phi\left(\rho_{y}<\theta, \theta\right)<0, \phi\left(\rho_{y}\right.$ $>\theta, \theta)>0$, and $\phi(0, \theta)=0$. Our Equation 6 can satisfy this condition if $A_{3}^{-}=0$, as is the case for our minimal triplet models. The second requirement is that the threshold $\theta$ between potentiation and depression is proportional to the expectation of the $p^{\text {th }}$ power of the postsynaptic firing rate, i.e., $\theta=\alpha\left\langle\rho_{y}^{p}\right\rangle$, where $p>1$ (Bienenstock et al., 1982; Intrator and Cooper, 1992). This second requirement can be fulfilled if the parameters $A_{2}^{-}$and $A_{2}^{+}$ depend on the mean firing rate $\left\langle\rho_{y}\right\rangle$ (or powers thereof) of the postsynaptic neuron. Specifically, we set $A_{2}^{-} \rightarrow A_{2}^{-}\left\langle\rho_{y}^{p}\right\rangle / \rho_{0}^{p}$ as well as $A_{2}^{+} \rightarrow A_{2}^{+}\left\langle\rho_{y}^{p}\right\rangle / \rho_{0}^{p}$. By doing so, the threshold becomes $\theta=$ $\left\langle\rho_{y}^{p}\right\rangle\left(A_{2}^{-} \tau_{-} A_{2}^{+} \tau_{+}\right) /\left(\rho_{0}^{p} A_{3}^{+} \tau_{+} \tau_{y}\right)$.

Strictly speaking, $\left\langle\rho_{y}^{p}\right\rangle$ corresponds to the expectation over the input statistics of the $p^{\text {th }}$ power of the postsynaptic firing rate. Practically, this quantity can be evaluated on-line by low-pass filtering $\rho_{y}^{p}$ with a time constant of the order of 10 min or more. With this range of time scale, $\left\langle\rho_{y}^{p}\right\rangle$ can be considered as constant (i.e., $\left\langle\rho_{y}^{p}\right\rangle \simeq \rho_{0}^{p}$ ) over the duration of the pairing, triplet, and 
quadruplet protocols we used in this study. As an aside, we note that with Nearest-Spike interactions, our triplet learning rule can almost (but not strictly) be mapped to a BCM learning rule.

Because the triplet rule shares properties with BCM theory, we expect that it generates input selectivity if a neuron receives a large number of inputs. Development of input selectivity is thought be an important property to account for receptive fields development.

For a numerical illustration of the input selectivity property of the triplet learning rule, we simulated the following scenario. We assume that our model neuron receives 100 afferents $(1 \leq i \leq$ 100), which are stimulated with Gaussian profiles $\nu_{i}=1 \mathrm{~Hz}+50$ $\mathrm{Hz} \exp \left[-(i-\mu)^{2} /\left(2 \times 10^{2}\right)\right], i=1, \ldots, 100$, the center $\mu$ of which is shifted randomly every $200 \mathrm{~ms}$ (Fig. 7C) over 10 possible positions. Presynaptic spikes are generated at time $t_{i}^{f}$ with a rate $\nu_{i}$. Each presynaptic spike generates an exponential postsynaptic potential with decay time constant $\tau=10 \mathrm{~ms}$, so that the total potential is $u=\Sigma_{i} \Sigma_{t_{i}^{f}<t} w_{i} \varepsilon_{0} \exp \left[-\left(t-t_{i}^{f}\right) / \tau\right]$, where $\varepsilon_{0}=1 \mathrm{mV}$. The postsynaptic firing rate increases with the membrane potential according to $\nu^{\text {post }}=1 \mathrm{~Hz}+g \times u$, where $g=10 \mathrm{~Hz} / \mathrm{mV}$. The neuron is stimulated over $60 \mathrm{~s}$, whereas synapses change according to our triplet learning rule. As we can see in Figure $7 D$, the neuron becomes automatically specialized to one of the 10 input patterns (i.e., the one with $\mu=70$ ). In other words, learning leads to input selectivity, a necessary property for receptive field development.

It is interesting to note that the dynamics of Equation 6 can be seen as a gradient ascent of an objective function $L$ (i.e., $\Delta w \alpha$ $\partial L / \partial w)$. Let $p=2$ and $\beta=A_{3}^{+} \tau_{+} \tau_{y} . L$ can be written as $L=$ $(\beta / 3) \rho_{y}^{3}-(\beta / \alpha) \theta^{2}$. If the model neuron has only two input afferents and, hence, only two synapses, this objective function $L$ (or energy landscape) (Fig. 7B) elicits two selective points, which correspond to the two maxima of the function $L$. The first maximum is at $w_{1}=1$ and $w_{2}=0$, and the second is at $w_{1}=0$ and $w_{2}$ $=1$. Therefore, the pattern of synaptic weight corresponds to input selectivity (i.e., the neuron is sensitive to only one of two inputs). Thus, the objective function can be used for a mathematical demonstration of input selectivity. Note that the objective function exists only if we assume that $\phi$ is a function of $\left\langle\rho_{y}^{p}\right\rangle$ and not $\left\langle\rho_{y}\right\rangle^{p}$ (See also Cooper et al., 2004).

\section{Discussion}

In this paper, we first showed the limitations of the standard pair-based STDP models in terms of predicting the outcome of several spike timing-based protocols. We then showed that a triplet learning rule is more suitable to reproduce those experimental protocols, namely, the frequency dependence of the pairing protocol as well as the triplet and quadruplet protocols. Finally, we showed the link between our triplet learning rule and the BCM learning rule. We found noteworthy and somewhat unexpected that our detailed modeling of frequency dependence of pairbased protocols and asymmetries in triplet protocols should lead under the assumption of Poisson spike trains to a known theoretical rule with well characterized features.

Throughout this paper, we compared the All-to-All interactions versus the Nearest-Spike interactions for pair-based models as well as for triplet-based models. Although Nearest-Spike interactions induce some potentially interesting nonlinearities in pairbased models (van Rossum et al., 2000; Izhikevich and Desai, 2003; Burkitt et al., 2004) (especially in the Poisson protocol), it is not possible to make a strict mapping of Nearest-Spike interactions models to the BCM rule, and more importantly pair-based models with Nearest-Spike interactions fail to reproduce the cor- rect frequency dependence in the pairing protocol as well as triplet and quadruplet experiments.

\section{Limitations}

Even if our triplet model can capture most of the triplet and quadruplet experiments, it is necessary to keep in mind the kind of experiments this model cannot reproduce. Because our model predicts weight changes as a function of the spike timing only, it fails to make any kind of inference for experiments that trigger explicitly other biophysical parameters such as $\mathrm{Ca}^{2+}$ concentration or postsynaptic membrane potential. We nevertheless think that this approach is interesting, because in one way or another, those biophysical parameters depend on the timing of the presynaptic and postsynaptic spikes. For example, the calcium concentration depends on the timing of the postsynaptic spike (via the back-propagating action potential) and the presynaptic spike (via voltage-gated calcium channels and NMDA channels).

Recent experiments (Froemke et al., 2005) show that the shape of the depression part of the learning window depends on the position of the synapse on the dendritic tree. Although our model does not include such geometrical properties, it is possible to account for the position of the synapse by changing explicitly the time constant $\tau_{-}$(characterizing the LTD part of the learning window) as a function of the distance between the synapse and the soma.

Even in the context of typical STDP experiments, some aspects are not covered by our model. In most STDP experiments, plasticity is induced after a repetition of a fixed number of pairs of presynaptic and postsynaptic spikes. Clearly, the amount of plasticity depends on the number of pairs. In fact, the amount of potentiation increases with the number of pairs of presynaptic and postsynaptic spikes and saturates at a given value (Senn et al., 2001; Froemke et al., 2006). This saturation is not taken into account in our present model, because the weight dependence is not explicitly mentioned. The dependence on the weights can easily be added in the triplet models (the parameters $A_{2}^{+}, A_{2}^{-}, A_{3}^{+}$, and $A_{3}^{-}$could also depend on $w$ ). Even if we have some indications (Bi and Poo, 2001; Wang et al., 2005) of how synapses change as a function of $w$, more experimental data are clearly needed to determine the correct weight dependence. It should be noted that if we add the dependence on the weight, there would not be an unambiguous mapping to the BCM theory.

\section{Alternative interpretations of the experimental data}

The goal of this study was to go as far as possible in the prediction of the weight change with only spike timing and no other neuronal variables or mechanism. It is interesting to note that our triplet learning rule can reproduce both the that have been explained by a postsynaptic potential effect (Sjöström et al., 2001) or by a suppression effect (Wang et al., 2005). In Sjöström's experiment, the increased potentiation at high frequency is explained by the increased membrane potential because of the accumulation of presynaptic inputs, whereas in our model the increased potentiation is attributable to the increase of the postsynaptic variable $o_{2}$. Combined with a suitable neuron model, an increased frequency would of course yield a higher potential on average. Wang et al. (2005) interpreted their triplet and quadruplet experiment as a result of a suppression mechanism (i.e., if a pre-post pair is followed by a post-pre pair, the latter depression term suppresses the first potentiation term, and not the other way round). This phenomenon is captured in our framework by the extra potentiation attributable to the 1-pre-2post triplet term. 


\section{Expansion perspective}

It is possible to see pair terms and triplet terms of Equations 3 and 4 in a more general framework (Gerstner and Kistler, 2002). From the point of view of pure spike timing-dependent plasticity, the instantaneous weight change is an unknown functional of the presynaptic spike train $X$ and the postsynaptic spike train $Y$ (i.e., $d w / d t=H[X, Y])$. In this framework, a pair-based STDP learning rule corresponds to the Volterra expansion of $H$ to the second order. In this paper, we pushed the expansion to the third order and showed that the prediction power increased a lot with only one or two extra parameters.

It is interesting to note that quadruplets data can be fitted with a triplet rule. This suggests that third-order terms (triplet terms) are good enough, and therefore there is no need to take into account higher-order terms. It is of course possible that new experiments will show the limitations of a triplet model and force us to consider higher-order terms. Clearly, the relevance of such an approach depends on how far we have to go in the expansion.

The learning rule as it is now does not depend directly on the membrane potential and therefore cannot reproduce the experiments of Sjöström et al. (2001) in which the membrane potential is controlled by the experimentalist. However, we should note that in the framework of the Volterra expansion of the unknown functional $H$, it is possible to assume that $H$ depends explicitly on the membrane potential (Gerstner and Kistler, 2002) and therefore captures the voltage dependence experiments of Sjöström et al. (2001).

\section{Comparison to other models}

It is interesting to note that our triplet learning rule has some similarities with the Senn-Markram-Tsodyks (SMT) model (Senn et al., 2001). In their model, they need a first presynaptic spike to activate a fraction of NMDA channels and then a postsynaptic spike to set some secondary messengers in an upstate and finally a last postsynaptic spike to trigger synaptic potentiation. Thus, their rule essentially consists of a triplet prepost-post term for potentiation and a triplet post-pre-pre term for depression. Even if their model makes implicit use of triplet terms, it should be noted that the order of spikes is different compared with our present triplet model. In our present triplet model, we need one presynaptic and two postsynaptic spikes regardless of the order (i.e., it encompasses pre-post-post as well as post-pre-post triplets of spikes). This difference is of particular importance if we want to fit the post-pre-post triplet experiments or quadruplet experiments of Wang et al. (2005). For those protocols, the SMT model cannot reproduce the data.

Another model that takes into account a multispike interaction is the Froemke-Dan learning rule (Froemke and Dan, 2002). Their model (which is in fact a quadruplet model) predicts a synaptic behavior that is in direct contrast with the synaptic dynamics given by Equations 3 and 4. In their model, if a postsynaptic spike precedes a pre-post pair of spikes, the effective potentiation will decrease as soon as the two postsynaptic spikes get closer to each other, whereas in our model, the opposite occurs. This is the main reason why the Froemke-Dan model, under a Poisson assumption for the presynaptic and postsynaptic spike trains, predicts an increasing depression as the postsynaptic firing rate increases, as reported by Izhikevich and Desai (2003), which seems unplausible in view of the results in Figure $8 C$ of $S j$ öström et al. (2001). It should be noted that the revised suppression model of Froemke et al. (2006) gets around this problem by setting two different saturation values: one for depression and one for potentiation.

Until now, we have not made any assumption about the cel- lular processes that are described by our triplet learning rule. It is known that the amount of potentiation or depression expressed by a synapse depends critically on the concentration of calcium. Moreover, we know that there is a supralinear summation of calcium on the postsynaptic site when an EPSP precedes an action potential (Waters et al., 2003). From this point of view, two closely spaced postsynaptic spikes can increase the level of calcium and therefore increase potentiation. This would correspond to the 1-pre-2-post triplet term of our formalism.

In this study, we showed that a minimal triplet model can capture most, but not all, aspects of the pairing, triplet, and quadruplet experiments. A natural extension of this study could be to include explicitly the dependence on biophysical quantities such as the $\mathrm{Ca}^{2+}$ concentration, the postsynaptic membrane potential, or other neuronal quantities. A more appealing approach would be to consider existing detailed biophysical models of synaptic plasticity and try to reduce them to a triplet model and therefore identify the underlying biological quantities of our triplet model.

\section{References}

Abarbanel H, Huerta R, Rabinovich M (2002) Dynamical model of longterm synaptic plasticity. Proc Natl Acad Sci USA 59:10137-10143.

Artola A, Bröcher S, Singer W (1990) Different voltage dependent thresholds for inducing long-term depression and long-term potentiation in slices of rat visual cortex. Nature 347:69-72.

Bi G, Poo M (1998) Synaptic modifications in cultured hippocampal neurons: dependence on spike timing, synaptic strength, and postsynaptic cell type. J Neurosci 18:10464-10472.

Bi G, Poo M (2001) Synaptic modification of correlated activity: Hebb's postulate revisited. Annu Rev Neurosci 24:139-166.

Bi G, Wang H (2002) Temporal asymmetry in spike timing-dependent synaptic plasticity. Physiol Behav 77:551-555.

Bi GQ (2002) Spatiotemporal specificity of synaptic plasticity: cellular rules and mechanisms. Biol Cybern 87:319-332.

Bienenstock E, Cooper L, Munro P (1982) Theory of the development of neuron selectivity: orientation specificity and binocular interaction in visual cortex. J Neurosci 2:32-48.

Burkitt AN, Meffin MH, Grayden D (2004) Spike-timing-dependent plasticity: the relationship to rate-based learning for models with weight dynamics determined by a stable fixed point. Neural Comput 16:885-940.

Cooper LN, Intrator N, Blais BS, Shouval HZ (2004) Theory of cortical plasticity, Chap 3. Singapore: World Scientific.

Dudek SM, Bear MF (1992) Homosynaptic long-term depression in area cal of hippocampus and effects of $N$-methyl-D-aspartate receptor blockade. Proc Natl Acad Sci USA 89:4363-4367.

Froemke R, Dan Y (2002) Spike-timing dependent plasticity induced by natural spike trains. Nature 416:433-438.

Froemke R, Poo MM, Dan Y (2005) Spike-timing-dependent synaptic plasticity depends on dendritic location. Nature 434:221-225.

Froemke R, Tsay I, Raad M, Long J, Dan Y (2006) Contribution of individual spikes in burst-induced long-term synaptic modification. J Neurophysiol 95:1620-1629.

Gerstner W, Kempter R, van Hemmen JL, Wagner H (1996) A neuronal learning rule for sub-millisecond temporal coding. Nature 383:76-78.

Gerstner W, Kistler WK (2002) Spiking neuron models. Cambridge: Cambridge UP.

Intrator N, Cooper LN (1992) Objective function formulation of the bcm theory of visual cortical plasticity. Neural Netw 5:3-17.

Izhikevich E, Desai N (2003) Relating stdp to bcm. Neural Comput 15:1511-1523.

Karmarkar U, Buonomano D (2002) A model of spike-timing dependent plasticity: one or two coincidence detectors. J Neurophysiology 88:507-513.

Kempter R, Gerstner W, van Hemmen JL (1999) Hebbian learning and spiking neurons. Phys Rev E 59:4498-4514.

Kirkwood A, Rioult MG, Bear MF (1996) Experience-dependent modification of synaptic plasticity in visual cortex. Nature 381:526-528.

Kistler WM, van Hemmen JL (2000) Modeling synaptic plasticity in con- 
junction with the timing of pre- and postsynaptic potentials. Neural Comput 12:385-405.

Lisman J (1989) A mechanism for hebb and anti-hebb processes underlying learning and memory. Proc Natl Acad Sci USA 86:9574-9578.

Lisman J, Spruston N (2005) Postsynaptic depolarization requirements for LTP and LTD: a critique of spike timing-dependent plasticity. Nature Neurosci 8:839-841.

Lisman J, Zhabotinsky A (2001) A model of synaptic memory: a CaMKII/ PP1 switch that potentiates transmission by organizing an AMPA receptor anchoring assembly. Neuron 31:191-201.

Malenka RC, Kauer J, Zucker R, Nicoll RA (1988) Postsynaptic calcium is sufficient for potentiation of hippocampal synaptic transmission. Science 242:81-84.

Markram H, Lübke J, Frotscher M, Sakmann B (1997) Regulation of synaptic efficacy by coincidence of postsynaptic AP and EPSP. Science 275:213-215.

Pfister JP, Gerstner W (2006) Beyond pair-based stdp: a phenomenologicalrule for spike triplet and frequency effects. In: Advances in neural information processing systems, Chap 18 (Weiss Y, Schölkopf B, Platt J, eds), pp 1083-1090. Cambridge, MA: MIT.

Rao RPN, Sejnowski TJ (2001) Spike-timing dependent Hebbian plasticity as temporal difference learning. Neural Comput 13:2221-2237.
Senn W, Markram H, Tsodyks M (2001) An algorithm for modifying neurotransmitter release probability based on pre- and postsynaptic spike timing. Neural Comput 13:35-67.

Shouval HZ, Bear MF, Cooper LN (2002) A unified model of NMDA receptor dependent bidirectional synaptic plasticity. Proc Natl Acad Sci USA 99:10831-10836.

Sjöström P, Turrigiano G, Nelson S (2001) Rate, timing, and cooperativity jointly determine cortical synaptic plasticity. Neuron 32:1149-1164.

Song S, Miller K, Abbott L (2000) Competitive Hebbian learning through spike-time-dependent synaptic plasticity. Nat Neurosci 3:919-926.

van Rossum MCW, Bi GQ, Turrigiano GG (2000) Stable Hebbian learning from spike timing-dependent plasticity. J Neurosci 20:8812-8821.

Wang HX, Gerkin RC, Nauen DW, Bi GQ (2005) Coactivation and timingdependent integration of synaptic potentiation and depression. Nat Neurosci 8:187-193.

Waters J, Larkum M, Sakmann B, Helmchen F (2003) Supralinear $\mathrm{CA}^{2+}$ influx into dendritic tufts of layer $2 / 3$ neocortical pyramidal neurons in vitro and in vivo. J Neurosci 23:8558-8567.

Zhang L, Tao H, Holt C, Harris W, Poo M-M (1998) A critical window for cooperation and competition among developing retinotectal synapses. Nature 395:37-44. 